\title{
Fields Nonlocal in Clifford Space. I. Classical Gauge-Invariant Nonlinear Field Theory*
}

\author{
Michael Danos \\ National Bureau of Standards, Washington, D. C. 20234 \\ and \\ Walter Greiner and Johann Rafelski \\ Institut für Theoretische Physik der Universität, Frankfurt am Main, Germany \\ (Received 31 July 1972)
}

\begin{abstract}
A fully gauge-invariant, Lorentz-covariant, nonlocal, and nonlinear theory, for ccupled spin- $\frac{1}{2}$ fields, $\psi$, and vector fields, $A$, i.e., "electrons" and "photons," is constructed. The field theory is linear in the $\psi$ fields. The nonlinearity in the $A$ fields arises unambiguously from the requirement of gauge invariance. The coordinates are generalized to admit hypercomplex values, i.e., they are taken to be Clifford numbers. The nonlocality is limited to the hypercomplex component of the coordinates. As the size of the nonlocality is reduced toward zero, the theory goes over into the inhomogeneous Dirac theory. The nonlocality parameter corresponds to an inverse mass and induces self-regulatory properties of the propagators. It is argued that in a gauge-invariant theory a graph-by-graph convergence is impossible in principle, but it is possible that convergence may hold for the complete solution, or for sums over classes of graphs.
\end{abstract}

\section{INTRODUCTION}

Recently the study of nonlinear field theories has gained increasing attention as the evidence has mounted concerning the convergence difficulties of conventional field theories. As is well known, the conventional theories contain linear "free-particle" parts; the nonlinearities enter only via the interaction Lagrangian. For example, the interaction is of the order 3 in fermion-boson fields, and of order 4 in the terms describing the interaction between different fermion fields. Both local and nonlocal interactions have been employed. The first departures from such minimally nonlinear theories were undertaken by adding next-higherorder terms to the Lagrangian. Examples of this approach are the Heisenberg theory, ${ }^{1}$ which adds a $(\bar{\psi} \psi)^{2}$ term, and the chiral theories which add $\varphi^{4}$ terms. ${ }^{2}$ In the newer efforts such piecemeal enlargements of the theories were abandoned in favor of the introduction into the interaction Lagrangians of massive nonlinearities, i.e., of series of terms up to infinite order in the fields, which are formally written as functions of fields. Both algebraic and transcendental functions have been employed. A relatively recent survey of these developments is given in Ref. 3 .

In attempting to construct a convergent field theory perhaps the most obvious and, unfortunately, unsuccessful choice is the introduction of cutoff form factors in the vertices of the interaction Lagrangian. As is well known, the reasons for this failure are that the form factors must contain re- tardation because of the requirement of Lorentz invariance, and thus upon quantization are simply equivalent to the introduction of an intermediate boson field. This intermediate boson field now is beset by all the divergences of the original field theory.

One now is left with the other possibility which is to change the form of the propagators. To achieve this one must modify the free-field Lagrangian rather than the interaction Lagrangian. This is the guiding thought of the present work. Our program thus is the following: Modify the free-field Lagrangian, and change the interaction Lagrangian only to such an extent as required by Lorentz and gauge invariance. This way all invariance requirements are built into the theory $a b$ initio; the theory is manifestly covariant throughout.

In the present paper we shall describe the field theory of a charged fermion field, $\psi$, interacting with a neutral vector-boson field, $A_{\mu}$. Upon quantization this would represent, for the case of a massless boson field, a generalization of quantum electrodynamics (QED). In this work we will try to maintain linearity in the $\psi$ field, i.e., the fermion field, and allow infinite order of the freefield operators. Then, as a consequence of charge conservation, one is forced to introduce infiniteorder nonlinearity in the photon field, i.e., the $A$ field. This way one obtains equations of motion which are formally linear in the $\psi$ field; a massive nonlinearity of the $\psi$ field arises only indirectly via the interaction with the $A$ field. Because of the 
appearance of infinite-order derivatives it is advantageous to write the fields as functions of hypercomplex numbers, ${ }^{4}$ i.e., to enlarge the Minkowski space of the coordinates to a Clifford-Minkowski space. As we shall see, this not only allows us to write formulas in a concise form, but, more importantly, turns out to be a powerful tool in the mathematical manipulations needed in deriving the results, i.e., in demonstrating the physical content of the particular field theory under investigation.

The paper is organized as follows. In Sec. II we introduce the Lagrangian of the noninteracting $\psi$ field giving a certain justification for the ansatz of the particular Lagrangian on which we concentrate in this paper. We then derive the free-field solutions and describe their properties. Finally, we determine expressions for the conserved quantities of this theory. In Sec. III expressions are given for the Feynman propagator of the theory. We do this in order to elucidate certain properties of the infinite-order equations of motion of the $\psi$ field. The interaction of the fermion field with the photon field in a manifestly gauge-invariant manner is achieved in Sec. IV by means of minimal coupling, i.e., by the substitution of $p \rightarrow p-e A$ in the free- $\psi$ field Lagrangian. This way we ensure charge conservation of the $\psi$ field. By use of Clifford algebra a very concise form for the field equations is derived and the gauge invariance of the obtained equations is explicitly verified. We demonstrate how certain possible generalizations of the linear field theory can be obtained from our field theory by an inconsistent limiting process, and this way we elucidate the reasons for the violation of charge conservation inherent in those theories. Finally, we also give a closed expression for the current vector in the presence of the $A$ field. In the concluding Sec. V our results are summarized and an outlook is given on the further developments of the theory implied by the results of the present paper.

\section{LINEAR FREE-FIELD EQUATIONS NONLOCAL IN CLIFFORD SPACE}

We propose in this section a theory for spin- $\frac{1}{2}$ particles with nonlocality in Clifford space. This means that the value of the field variables $\psi$ at a point $x_{\mu}$ will be connected by the field equations with the value of $\psi$ at a point $x_{\mu}+\gamma_{\mu} h$. The hypercomplex numbers $\gamma_{\mu}$, i.e., the Clifford numbers, are in fact the Dirac $\gamma$ matrices with $\left[\gamma_{\mu}, \gamma_{\nu}\right]=2 g_{\mu \nu}$. (We use Bjorken-Drell conventions. ${ }^{5}$ ) This nonlocality is defined by the Taylor series ${ }^{8}$

$$
\begin{aligned}
\psi\left(x_{\mu}+\gamma_{\mu} h\right) & =\sum_{n=0}^{\infty} \frac{\left(h \gamma_{\nu} \partial^{v}\right)^{n}}{n !} \psi\left(x_{\mu}\right) \\
& =\psi\left(x_{\mu}\right)+h \gamma_{\nu} \partial^{v} \psi\left(x_{\mu}\right)+\cdots .
\end{aligned}
$$

In this expansion odd and even powers of the Lorentz scalar $\gamma_{\mu} \partial^{\mu} \equiv \gamma \cdot \partial$ occur. Even powers have Dirac unit-matrix operator properties because $\gamma_{\mu} \partial^{\mu} \gamma_{\nu} \partial^{\nu}=\partial_{\mu} \partial^{\mu} \equiv \square$. Thus even powers of $(\gamma \partial)$ are associated with spin- 0 particles and odd powers with spin- $\frac{1}{2}$ particles. In order to separate the properties of spin- 0 and spin- $\frac{1}{2}$ particles we consider the following linear combinations of nonlocalities:

$$
\begin{aligned}
& \psi\left(x_{\mu}+\gamma_{\mu} h\right)-\psi\left(x_{\mu}-\gamma_{\mu} h\right)=2 \sum_{n=0}^{\infty} \frac{(h \gamma \cdot \partial)^{2 n+1}}{(2 n+1) !} \psi\left(x_{\mu}\right), \\
& \varphi\left(x_{\mu}+\gamma_{\mu} h\right)+\varphi\left(x_{\mu}-\gamma_{\mu} h\right)=2 \sum_{n=0}^{\infty} \frac{(h \gamma \cdot \partial)^{2 n}}{(2 n) !} \varphi\left(x_{\mu}\right)
\end{aligned}
$$

where Eq. (2a) is suited for fermions and Eq. (2b) for bosons. Using the properties of the $\gamma_{\mu}$ matrices and observing that symmetrization of the $\gamma_{\mu} \partial^{\mu}$ products is not necessary, we obtain

$$
\begin{aligned}
& \psi(x+\gamma h)-\psi(x-\gamma h)=2 \sum_{n=0}^{\infty} \frac{h^{2 n}}{(2 n+1) !} \square^{n}(h \gamma \cdot \partial) \psi(x), \\
& \varphi(x+\gamma h)+\varphi(x-\gamma h)=2 \sum_{n=0}^{\infty} \frac{h^{2 n}}{(2 n) !} \square^{n} \varphi(x) .
\end{aligned}
$$

Upon inclusion of appropriate factors Eq. (3a) is a possible generalization of the Dirac operator $(i \gamma \partial)$ and Eq. (3b) a possible generalization of the KleinGordon operator $\square$. For spin- $\frac{1}{2}$ particles it is explicitly

$$
\begin{aligned}
\frac{i}{2 h}[\psi(x+\gamma h)-\psi(x-\gamma h)] & =\sum_{n=0}^{\infty} \frac{h^{2 n}}{(2 n+1) !} \square^{n}(i \gamma \cdot \partial) \psi(x) \\
& \underset{h \rightarrow 0}{\longrightarrow}(i \gamma \cdot \partial) \psi(x) .
\end{aligned}
$$

$|h|$ has a meaning of a small "elementary" length; thus $(h \gamma \partial)$ is dimensionless, and transforms like a scalar under Lorentz transformations. The value of $|h|$ should be sufficiently small, so that no contradictions arise with known experiments. $1 /|h|$ corresponds to a "cutoff" mass and may be very large, for example larger than, say, $100 \mathrm{GeV}$.

The free-particle spin- $\frac{1}{2}$ equation now reads

$$
\frac{i}{2 h}[\psi(x+\gamma h)-\psi(x-\gamma h)]-m \psi(x)=0 .
$$

Similar arguments may be used to generalize the Klein-Gordon equation. This way one obtains from (3b) 


$$
h^{-2}[\varphi(x+\gamma h)+\varphi(x-\gamma h)-2 \varphi(x)]-m^{2} \varphi(x)=0 .
$$

It is understood, that the $\gamma_{\mu}$ matrix of the argument of the $\psi$ functions operates on $\psi$, as defined by the Taylor expansion Eq. (3a). That is, we should write $(x+\gamma h) \psi$ rather than $\psi(x+\gamma h)$. Nevertheless, we shall use the notation of Eq. (5) since no ambiguities will arise.

The above equation is a functional equation and is derivable from a variational principle. Before demonstrating this, let us investigate a more general case of a nonlocal Lagrangian,

$$
\mathscr{L}=\mathscr{L}(\varphi(x), \varphi(x+a), \varphi(x-a)) .
$$

Using Hamilton's principle

$$
\delta \int_{S} d^{4} x \mathfrak{L}=0
$$

we obtain, if, as usual, the functions are not varied at the boundary of the support $S$ of Eq. (7), the following equation:

$$
\begin{aligned}
0 & =\delta \int_{S} d^{4} x \mathfrak{L} \\
& =\int_{S} d^{4} x \delta \mathfrak{L} \\
& =\int_{S} d^{4} x\left(\frac{\partial \mathcal{L}}{\partial \varphi(x)} \delta \varphi(x)+\frac{\partial \mathcal{L}}{\partial \varphi(x+a)} \delta \varphi(x+a)+\frac{\partial \mathcal{L}}{\partial \varphi(x-a)} \delta \varphi(x-a)\right) .
\end{aligned}
$$

We find an expression for $\delta \varphi(x+a)$ using the fact that a formal Taylor expansion is possible and that $\delta$ commutes with the differentiation operators,

$$
\delta \varphi(x+a)=\sum_{n=0}^{\infty} \frac{1}{n !}\left(a_{\mu} \partial^{\mu}\right)^{n} \delta \varphi(x) .
$$

We integrate Eq. (8a) by parts and drop the surface terms. The result is

$$
0=\int_{S} d^{4} x\left(\frac{\partial \mathcal{L}}{\partial \varphi(x)}+\sum_{n=0}^{\infty} \frac{(-)^{n}}{n !}\left(a_{\mu} \partial^{\mu}\right)^{n} \frac{\partial \mathcal{L}}{\partial \varphi(x+a)}+\sum_{n=0}^{\infty} \frac{(-)^{n}}{n !}\left(-a_{\mu} \partial^{\mu}\right)^{n} \frac{\partial \mathscr{L}}{\partial \varphi(x-a)}\right) \delta \varphi(x) .
$$

The operator $\sum\left(-a_{\mu} \partial^{\mu}\right)^{n} / n$ ! is a linear displacement operator; it translates in Eq. (9) the function

$$
\partial \mathcal{L}(\varphi(x), \varphi(x+a), \varphi(x-a)) / \partial \varphi(x+a)
$$

from the point $x$ to the point $x-a$. We thus obtain

$$
0=\int_{S} d^{4} x\left(\frac{\partial \mathcal{L}(\varphi(x), \varphi(x+a), \varphi(x-a))}{\partial \varphi(x)}+\frac{\partial \mathcal{L}(\varphi(x-a), \varphi(x), \varphi(x-2 a))}{\partial \varphi(x)}+\frac{\partial \mathcal{L}(\varphi(x+a), \varphi(x+2 a), \varphi(x))}{\partial \varphi(x)}\right) \delta \varphi(x),
$$

that is,

$$
0=\int_{S} d^{4} x \delta \varphi(x) \frac{\partial}{\partial \varphi(x)}[\mathcal{L}(x)+\mathcal{L}(x-a)+\mathcal{L}(x+a)] .
$$

The Euler-Lagrange equation now reads

$$
0=\frac{\partial}{\partial \varphi(x)}[\mathfrak{L}(x)+\mathscr{L}(x-a)+\mathscr{L}(x+a)] .
$$

Equation (11) can be derived directly from Eq. (8) if the support $S \pm a$ is taken to be larger than the support $S$ of $\mathcal{L}$ [this assumption is equivalent to the assumption that surface terms can be neglected in derivation of Eq. (9)].

The variation of the Lagrange function is

$$
\begin{aligned}
0 & =\int_{S} d^{4} x \delta \mathcal{L} \\
& =\int_{S} d^{4} x_{1} \frac{\partial \mathcal{L}\left(x_{1}\right)}{\partial \varphi\left(x_{1}\right)} \delta \varphi\left(x_{1}\right)+\int_{S} d^{4} x_{2} \frac{\partial \mathcal{L}\left(x_{2}\right)}{\partial \varphi\left(x_{2}+a\right)} \delta \varphi\left(x_{2}+a\right)+\int_{S} d^{4} x_{3} \frac{\partial \mathcal{L}\left(x_{3}\right)}{\partial \varphi\left(x_{3}-a\right)} \delta \varphi\left(x_{3}-a\right) .
\end{aligned}
$$


Setting $x=x_{2}+a, x=x_{3}-a, x=x_{1}$, we obtain

$$
\begin{aligned}
0 & =\int_{S} d^{4} x \delta \mathscr{L} \\
& =\int_{S} d^{4} x \frac{\partial \mathscr{L}(x)}{\partial \varphi(x)} \delta \varphi(x)+\int_{S-a} d^{4} x \frac{\partial \mathscr{L}(x-a)}{\partial \varphi(x)} \delta \varphi(x)+\int_{S+a} d^{4} x \frac{\partial \mathscr{L}(x+a)}{\partial \varphi(x)} \delta \varphi(x) .
\end{aligned}
$$

Now, following the assumption that the support of the integrals has the above-mentioned property,

$$
S \subset S-a, \quad S \subset S+a,
$$

we obtain (10b), where from the variational Eq. (11) follows immediately.

A generalization of the variational principle is obtained immediately for the case where $\mathscr{L}$ is not only a function of the field variables at different discrete points, but also a function of a continuous region of points, that is,

$$
\mathscr{L}=\mathscr{L}\left(\varphi(x), \varphi(x+a), \ldots, \int_{b}^{c} \varphi(x+y) d y\right) .
$$

We have

$$
\begin{aligned}
0 & =\int d^{4} x \delta \mathscr{L} \\
& =\int d^{4} x\left(\frac{\partial \mathcal{L}}{\partial \varphi(x)} \delta \varphi(x)+\frac{\partial \mathscr{L}}{\partial \varphi(x+a)} \delta \varphi(x+a)+\cdots+\int_{b}^{c} d y \frac{\partial \mathscr{L}}{\partial \varphi(x+y)} \delta \varphi(x+y)\right),
\end{aligned}
$$

and herefrom we obtain as in Eq. (10b)

$$
0=\int d^{4} x \delta \varphi(x) \frac{\partial}{\partial \varphi(x)}\left(\mathscr{L}(x)+\mathcal{L}(x-a)+\cdots+\int_{b}^{c} d y \frac{\partial \mathcal{L}(x-y)}{\partial \varphi(x)}\right),
$$

where

$$
\mathcal{L}(x-y)=\mathcal{L}(\varphi(x-y), \varphi(x+a-y), \ldots,(c-b) \varphi(x)) .
$$

The Euler-Lagrange equation (11) now reads in the generalized form

$$
0=\frac{\partial}{\partial \varphi(x)}\left(\sum_{k=1}^{N} \mathcal{L}\left(x-a_{k}\right)+\int_{b}^{c} d y \frac{\partial \mathscr{L}(x-y)}{\partial \varphi(x)}\right) .
$$

We now derive Eq. (5) (spin- $\frac{1}{2}$ equation),

$$
(i / 2 h)[\psi(x+\gamma h)-\psi(x-\gamma h)]-m \psi(x)=0,
$$
and its adjoint,

$$
\left(-i / 2 h^{*}\right)\left[\bar{\psi}\left(x+\gamma h^{*}\right)-\bar{\psi}\left(x-\gamma h^{*}\right)\right]-m \bar{\psi}(x)=0,
$$

from a Lagrangian, which we will define presently Let us first explain the adjoint wave function in Eq. (12) more thoroughly. We have $\left(\gamma^{\dagger}=\gamma_{0} \gamma \gamma_{0}\right)$ :

$$
\begin{aligned}
\overline{\psi(x+\gamma h)} & \equiv\left(\sum_{n=0}^{\infty} \frac{(h \gamma \cdot \partial)^{n}}{n !} \psi(x)\right)^{\dagger} \gamma_{0} \\
& =\left(\psi^{\dagger}(x) \sum \frac{\left(h^{*} \gamma^{\dagger} \cdot \bar{\partial}\right)^{n}}{n !}\right) \gamma_{0} \\
& =\psi^{\dagger}(x) \gamma_{0} \sum \frac{\left(h^{*} \gamma \cdot \bar{\partial}\right)^{n}}{n !} \\
& =\bar{\psi}(x) \sum \frac{\left(h^{*} \gamma \cdot \bar{\partial}\right)^{n}}{n !} \\
& =\bar{\psi}\left(x+\gamma h^{*}\right) .
\end{aligned}
$$

\section{Consider now the Lagrangian}

$$
\begin{aligned}
\mathscr{L}= & (i / 2 h) \bar{\psi}(x)[\psi(x+\gamma h)-\psi(x-\gamma h)] \\
& -m \bar{\psi}(x) \psi(x) .
\end{aligned}
$$

Equation (5) follows directly from $\delta \mathscr{L} / \delta \bar{\psi}=\partial \mathscr{L}(x) /$ $\partial \bar{\psi}(x)=0$. We calculate now $\delta \mathcal{L} / \delta \psi$ using Eq. (11). With $a=\gamma h$ this equation reads after straightforward calculation

$$
0=(-i / 2 h)[\bar{\psi}(x+\gamma h)-\bar{\psi}(x-\gamma h)]-m \bar{\psi}(x) .
$$

A comparison of (12) and (14) shows that there are two possible choices of $h$ :

$$
\begin{aligned}
& h=h^{*}, \\
& h=-h^{*},
\end{aligned}
$$

that is, $h$ must be either a real or an imaginary number when the Lagrangian (13) is used. As it is the simplest possible Lagrangian, the "principle of simplicity" causes this result. Another way to understand (15) is to note that the operator $(1 / h)$ $\sin h p$ [see Eq. (18)] would not be Hermitian otherwise. We investigate the theory with real $h$, as it will turn out that then all the poles of the Green's function lie on the real axis. In the Appendix we give a short discussion of the properties of the theory with imaginary $h$, where the poles lie in the complex $\omega$ plane.

Let us now introduce an operator notation which 
will be of great convenience later. The field equation (5) obviously can be written

$$
\frac{e^{-h \gamma \cdot \partial}-e^{h \gamma \cdot \partial}}{2 h i} \psi(x)-m \psi(x)=0
$$

where $\exp (h \gamma \cdot \partial)=\sum(h \gamma \cdot \partial)^{n} / n$ ! is the Taylor expansion operator. Equation (16) may be equivalently written

$$
(1 / h)(\sin i h \gamma \cdot \partial) \psi(x)-m \psi(x)=0 .
$$

Using $i \gamma_{\mu} \partial^{\mu}=\not p$ we obtain

$$
(1 / h)(\sinh \not) \psi(x)-m \psi(x)=0,
$$

or, formally,

$$
\frac{\sin (h \not p)}{h \not p} \not p \psi(x)-m \psi(x)=0
$$

with the operator

$$
\begin{aligned}
\frac{\sin (h \not p)}{h \not p} & =\sum_{n=0}^{\infty} \frac{h^{n}}{(2 n+1) !} \square^{n} \\
& =\prod_{k=1}^{\infty}\left(1+\frac{h^{2} \square}{k^{2} \pi^{2}}\right) .
\end{aligned}
$$

We now solve (17) for the case of plane waves, i.e., we set

$$
\begin{aligned}
& \psi^{r}(x)=w^{r}(p) e^{-i \epsilon_{r} p_{\mu} x^{\mu}}, \\
& r=1,2,3,4, \quad \epsilon_{r}= \begin{cases}+1 & \text { for } r=1,2 \\
-1 & \text { for } r=3,4\end{cases}
\end{aligned}
$$

Equation (17) then reads

$$
(1 / h) \sin \left(\epsilon_{r} h \gamma_{\mu} p^{\mu}\right) w^{r}(p)-m w^{r}(p)=0,
$$

or, written in analogy to Eq. (19), explicitly showing the matrix operators $\left(p^{2}=p_{\mu} p^{\mu}=\omega^{2}-k^{2}\right.$ is a number),

$$
\frac{\sin \left[h\left(p^{2}\right)^{1 / 2}\right]}{h\left(p^{2}\right)^{1 / 2}} \epsilon_{r} \not p w^{r}(p)-m w^{r}(p)=0 .
$$

Defining the numbers

$$
\Omega=m \frac{h\left(p^{2}\right)^{1 / 2}}{\sin \left[h\left(p^{2}\right)^{1 / 2}\right]},
$$

(22) can be written as

$$
\epsilon_{r} \not p w^{r}(p, \Omega)-\Omega w^{r}(p, \Omega)=0
$$

which is the usual Dirac problem.

We gain more insight about the kinematics of our new wave equation when we "square" Eq. (22). We obtain

$$
\left(1 / h^{2}\right) \sin ^{2}\left[h\left(p^{2}\right)^{1 / 2}\right]=m^{2}
$$

which yields with

$$
M_{0} h \equiv \arcsin (h m)
$$

the following solutions:

$$
\begin{aligned}
& h\left(p^{2}\right)^{1 / 2}=M_{0} h, \\
& h\left(p^{2}\right)^{1 / 2}=\pi n_{ \pm} M_{0} h, \quad n=1,2, \ldots .
\end{aligned}
$$

Note that because of $p^{2}=\omega^{2}-k^{2}$, we get

$$
\begin{aligned}
& \left(\omega^{2}-k^{2}\right)^{1 / 2}=M_{0}, \\
& \left(\omega^{2}-k^{2}\right)^{1 / 2}=M_{n}{ }^{+} \equiv(\pi n / h) \pm M_{0}, \quad n=1,2, \ldots .
\end{aligned}
$$

We see that the usual dynamics is not changed as the usual dispersion relation holds:

$$
\omega^{2}=\left(M_{n}^{ \pm}\right)^{2}+k^{2} .
$$

The only difference is that a discrete spectrum $M_{n}$ of masses appears. From (26) we have

$$
M_{0}=m+\frac{1}{6}(m h)^{2} m+\cdots \cdot
$$

This equation means that for the mass $M_{0}$ a finite mass renormalization is effected. For $n \neq 0$ a "cutoff" spectrum of masses has been found. Note also that the roots of Eq. (27) would become complex for $h m>1$.

When solving the plane-wave equation (22) we found that the solutions were usual Dirac plane waves with a mass defined by Eq. (23). Inserting (25) into (23) we obtain

$$
\Omega^{2}=p^{2} \text {. }
$$

This means that

$$
M_{n}^{ \pm}=\Omega
$$

and we found that the values of $\Omega$ are discrete. Therefore the solutions of Eq. (21) must bear an additional discrete quantum number $n$, which belongs to the mass spectrum. The solutions of Eq. (21) are then given (cf. Bjorken-Drell) by

$$
\left(w_{n}^{1}, w_{n}^{2}, w_{n}^{3}, w_{n}^{4}\right)=\left(\frac{E+M_{n}^{ \pm}}{2 M_{n}^{ \pm}}\right)^{1 / 2}\left(\begin{array}{cccc}
1 & 0 & p_{z} /\left(E+M_{n}^{ \pm}\right) & p_{-} /\left(E+M_{n}^{ \pm}\right) \\
0 & 1 & p_{+} /\left(E+M_{n}^{ \pm}\right) & -p_{z} /\left(E+M_{n}^{ \pm}\right) \\
p_{z} /\left(E+M_{n}^{ \pm}\right) & p_{-} /\left(E+M_{n}^{ \pm}\right) & 1 & 0 \\
p_{+} /\left(E+M_{n}^{ \pm}\right) & -p_{z} /\left(E+M_{n}^{ \pm}\right) & 0 & 1
\end{array}\right) .
$$

We now show that the free-spin- $\frac{1}{2}$ equation has divergence-free tensors which define differentially conserved quantities. We start with a divergence-free four-vector which thus can serve as current four-vector. As we possess a Lagrangian, we could derive it by means of a generalized Noether theorem. Much 
easier and more instructive for later developments is to construct the current vector from the wave equations (5) and (12). We multiply Eq. (5) by $\bar{\psi}(x)$ from the left and Eq. (12) by $\psi(x)$ from the right and write these equations using differential operators as in Eq. (17):

$$
\begin{aligned}
& \bar{\psi}(x) h^{-1} \sin (i h \gamma \cdot \vec{\partial}) \psi(x)-m \bar{\psi}(x) \psi(x)=0, \\
& \bar{\psi}(x) h^{-1} \sin (-i h \gamma \cdot \bar{\partial}) \psi(x)-m \bar{\psi}(x) \psi(x)=0 .
\end{aligned}
$$

The difference of both equations is

$$
0=\bar{\psi}(x) \sin (i h \gamma \cdot \vec{\partial}) \psi(x)+\bar{\psi}(x) \sin (i h \gamma \cdot \bar{\partial}) \psi(x),
$$

or, explicitly,

$$
0=\sum_{n=0}^{\infty} \frac{h^{2 n+1}}{(2 n+1) !} \bar{\psi}(x)\left[(\gamma \cdot \vec{\partial})^{2 n+1}+(\gamma \cdot \vec{\partial})^{2 n+1}\right] \psi(x) .
$$

Our task now is to express

$$
\bar{\psi}(x)\left[(\gamma \cdot \bar{\partial})^{2 n+1}+(\gamma \cdot \vec{\partial})^{2 n+1}\right] \psi(x)
$$

as a divergence of some operator. In the case $n=0$ which gives the usual Dirac current it is simply $\partial_{\mu}\left(\bar{\psi} \gamma^{\mu} \psi\right)$. In the theory of the Klein-Gordon wave equation the similar expression

$$
0=\varphi *\left(\vec{\partial}_{\mu} \overleftrightarrow{\partial}^{\mu}-\vec{\partial}_{\mu} \vec{\partial}^{\mu}\right) \varphi
$$

occurs. The divergence equation there is

$$
0=\partial_{\mu}\left[\varphi^{*}\left(\vec{\partial}^{\mu}-\vec{\partial}^{\mu}\right) \varphi\right] \text {. }
$$

In analogy we expect

$$
\bar{\psi}(x)\left[(\gamma \cdot \bar{\partial})^{2 n+1}+(\gamma \cdot \vec{\partial})^{2 n+1}\right] \psi(x)=\partial_{\mu} \sum_{k=0}^{2 n}(-)^{k}\left[\bar{\psi}(x)(\gamma \cdot \overleftarrow{\partial})^{k} \gamma^{\mu}(\gamma \cdot \vec{\partial})^{2 n-k} \psi(x)\right] .
$$

Simple differentiation proves this identity. Thus we obtain for the current after insertion of all necessary factors

$$
j_{\mu}=e \bar{\psi}(x) \overrightarrow{\partial_{\mu}} \psi(x)
$$

with

$$
\overrightarrow{\mathrm{J}}_{\mu}=\sum_{n=0}^{\infty} \sum_{k=0}^{2 n} \frac{(-)^{k} h^{2 n}}{(2 n+1) !}(\gamma \cdot \vec{\partial})^{2 n-k} \gamma_{\mu}(\gamma \cdot \vec{\partial})^{k}
$$

For $h \rightarrow 0$ this expression reduces to the usual $e \bar{\psi} \gamma_{\mu} \psi$.

The energy-momentum tensor in the Dirac theory is given by

$$
T^{\mu v}=-\frac{1}{2} i \bar{\psi}\left(\gamma^{\mu} \vec{\partial}^{\nu}-\gamma^{\mu} \vec{\partial}^{\nu}\right) \psi \text {. }
$$

This is a Hermitian, but not a symmetric tensor. Symmetrization is effected through terms like

$$
\bar{\psi}\left(\gamma^{\nu} \partial^{\mu}-\gamma^{\nu \partial^{\mu}}\right) \psi \text {. }
$$

In analogy to the current derived above we try the ansatz

$$
T^{\mu \nu}=-\frac{1}{2} i \bar{\psi}\left\{\sum_{n=0}^{\infty} \sum_{k=0}^{\infty} \frac{(-)^{k} h^{2 n}}{(2 n+1) !}(\gamma \cdot \vec{\partial})^{k}\left[\gamma^{\mu} \vec{\partial}^{\nu}-\gamma^{\mu} \partial^{\nu}\right](\gamma \cdot \vec{\partial})^{2 n-k}\right\} \psi .
$$

As this tensor is not symmetric we have to prove both divergence conditions:

$$
\partial_{\mu} T^{\mu \nu}=0, \quad \partial_{\nu} T^{\mu \nu}=0 .
$$

We begin with the first form: 


$$
\begin{aligned}
\partial_{\mu} T^{\mu \nu}= & -\frac{1}{2} i \Psi\left\{\sum_{n=0}^{\infty} \sum_{k=0}^{2 n} \frac{(-)^{k} h^{2 n}}{(2 n+1) !}(\gamma \cdot \overleftarrow{\partial})^{k}\left[\vec{\partial}_{\mu} \gamma^{\mu} \vec{\partial}^{\nu}+\vec{\partial}_{\mu} \gamma^{\mu} \vec{\partial}^{\nu}-\vec{\partial}_{\mu} \gamma^{\mu} \vec{\partial}^{\nu}-\vec{\partial}_{\mu} \gamma^{\mu} \vec{\partial}^{\nu}\right](\gamma \cdot \vec{\partial})^{2 n-k}\right\} \psi \\
= & -\frac{1}{2} i \bar{\psi}\left\{\sum_{n=0}^{\infty} \sum_{k=0}^{2 n} \frac{(-)^{k} h^{2 n}}{(2 n+1) !}(\gamma \cdot \vec{\partial})^{k+1}\left[\vec{\partial}^{\nu}-\bar{\partial}^{\nu}\right](\gamma \cdot \vec{\partial})^{2 n-k}\right\} \psi \\
& -\frac{1}{2} i \bar{\psi}\left\{\sum_{n=0}^{\infty} \sum_{k=0}^{2 n} \frac{(-)^{k} h^{2 n}}{(2 n+1) !}(\gamma \cdot \bar{\partial})^{k}\left[\vec{\partial}^{\nu}-\bar{\partial}^{\nu}\right](\gamma \cdot \vec{\partial})^{2 n-k+1}\right\} \psi .
\end{aligned}
$$

When adding the two terms on the right-hand side, only the term with $k=0$ in the second part of the total sum and the term $k=2 n$ in the first part of the total sum remain:

$$
\partial_{\mu} T^{\mu \nu}=-\frac{1}{2 h} i \bar{\psi}\left\{\sum_{n=0}^{\infty} \frac{h^{2 n+1}}{(2 n+1) !}(\gamma \cdot \vec{\partial})^{2 n+1}\left[\vec{\partial}^{\nu}-\overleftarrow{\partial}^{\nu}\right]\right\} \psi-\frac{1}{2 h} i \bar{\psi}\left\{\sum \frac{h^{2 n+1}}{(2 n+1) !}\left[\vec{\partial}^{v}-\overleftarrow{\partial}^{v}\right](\gamma \cdot \vec{\partial})^{2 n+1}\right\} \psi \cdot
$$

Using the wave equations (5) and (12) we finally obtain

$$
\begin{aligned}
\partial_{\mu} T^{\mu \nu} & =m \bar{\psi}\left[\vec{\partial}^{v}-\vec{\partial}^{\nu}\right] \psi-\bar{\psi}\left[\vec{\partial}^{\nu}-\vec{\partial}^{\nu}\right] m \psi \\
& =0 .
\end{aligned}
$$

We now prove the second part of Eq. (37):

$$
\partial_{\nu} T^{\mu \nu}=-\frac{1}{2} i \bar{\psi}\left\{\sum_{n=0}^{\infty} \sum_{k=0}^{2 n} \frac{(-)^{k} h^{2 n}}{(2 n+1) !}(\gamma \cdot \vec{\partial})^{k}\left[\vec{\partial}_{\nu} \gamma^{\mu} \vec{\partial}^{\nu}+\gamma^{\mu} \vec{\partial}_{\nu} \vec{\partial}^{\nu}-\gamma^{\mu} \overleftarrow{\partial}^{\nu} \overleftarrow{\partial}_{\nu}-\vec{\partial}^{\nu} \gamma^{\mu} \vec{\partial}_{\nu}\right](\gamma \cdot \vec{\partial})^{2 n-k}\right\} \psi .
$$

Only the middle terms remain:

$$
\{\cdots\}=\left\{\sum_{n=0}^{\infty} \sum_{k=0}^{2 n} \frac{(-)^{k} h^{2 u}}{(2 n+1) !}\left[(\gamma \cdot \grave{\partial})^{k} \gamma_{\mu}(\gamma \cdot \vec{\partial})^{2 n-k+2}-(\gamma \cdot \overleftarrow{\partial})^{k+2} \gamma_{\mu}(\gamma \cdot \vec{\partial})^{2 n-k}\right]\right\} \text {. }
$$

Again the sum over $k$ cancels out up to four terms: $k=0, k=1$ for the left term and $k=2 n, k=2 n-1$ for the right term. We obtain explicitly

$$
\partial_{\nu} T^{\mu \nu}=-\frac{1}{2} i \bar{\psi}\left\{\sum_{n=0}^{\infty} \frac{h^{2 n}}{(2 n+1) !}\left[\gamma^{\mu}(\gamma \cdot \vec{\partial})(\gamma \cdot \vec{\partial})^{2 n+1}-(\gamma \cdot \overleftarrow{\partial})^{2 n+1}(\gamma \cdot \vec{\partial}) \gamma^{\mu}-(\gamma \cdot \overleftarrow{\partial}) \gamma^{\mu}(\gamma \cdot \vec{\partial})^{2 n+1}+(\gamma \cdot \vec{\partial})^{2 n+1} \gamma^{\mu}(\gamma \cdot \vec{\partial})\right]\right\} \psi \cdot
$$

We can use the field equation as before and arrive at

$$
\begin{aligned}
\partial_{\nu} T^{\mu \nu} & =-\bar{\psi} \gamma_{\mu}(\gamma \cdot \vec{\partial}) m \psi-m \bar{\psi}(\gamma \cdot \bar{\partial}) \gamma_{\mu} \psi+\bar{\psi}(\gamma \cdot \bar{\partial}) \gamma_{\mu} m \psi+\bar{\psi} m \gamma_{\mu}(\gamma \cdot \vec{\partial}) \psi \\
& =0 .
\end{aligned}
$$

With this result the proof is completed that Eq. (36) indeed is divergence-free. As this expression reduces for $h \rightarrow 0$ to the Dirac energy-momentum tensor we expect it to be an appropriate form for our further investigations.

Our intuitive method in deriving $j_{\mu}$ and $T^{\mu \nu}$ may be made more understandable by simple means. Let us consider the expression (37) once more:

$$
0=f(x)[D(\gamma \cdot \overleftarrow{\partial})+D(\gamma \cdot \vec{\partial})] g(x) \text {. }
$$

Equation $\left(37^{\prime}\right)$ is a generalization of (37) in the sense that $f, g$, and $D$ are arbitrary. We may further write formally

$$
\begin{aligned}
0 & =f(x)\left[\left(\vec{\partial}_{\mu}+\overleftarrow{\partial}_{\mu}\right) J^{\mu}(\gamma \cdot \vec{\partial}, \gamma \cdot \overleftarrow{\partial})\right] g(x) \\
& =\partial_{\mu}\left[f(x) J^{\mu}(\gamma \cdot \vec{\partial}, \gamma \cdot \vec{\partial}) g(x)\right] .
\end{aligned}
$$

The characteristic function $J^{\mu}(\gamma a, \gamma b)$ is defined by the functional equation

$$
J^{\mu}(\gamma a, \gamma b)=\frac{D(\gamma a)+D(\gamma b)}{a_{\mu}+b_{\mu}} .
$$

Solutions of (38) in our special case are easily found, if we "forget" for a moment the matrix character of the involved functions:

$$
\begin{aligned}
\frac{D(a)+D(b)}{a+b} & =\frac{\sin (i h a)+\sin (i h b)}{a+b} \\
& =i \sum \frac{h^{2 n}}{(2 n+1) !} \frac{a^{2 n+1}+b^{2 n+1}}{a+b} \\
& =i \sum_{n=0}^{\infty} \frac{h^{2 n}}{(2 n+1) !} \sum_{k=0}^{2 n}(-)^{k} a^{k} b^{2 n-k} .
\end{aligned}
$$


We regain the matrix character using the apparent symmetry of Eq. (38):

$$
J^{\mu}\left(a_{\nu}, b_{\nu}\right)=J^{\mu}\left(b_{\nu}, a_{\nu}\right) .
$$

Equation (33) is symmetric in $a, b$. Replacing $a$ by $a_{\nu} \gamma^{\nu}$ and $b$ by $b_{\nu} \gamma^{\nu}$ we have to introduce $\gamma^{\mu}$ somewhere. Because of the above symmetry the only appropriate place is between $a^{k}$ and $b^{2 n-k}$; we obtain Eq. (35). A similar derivation is valid for the $T^{\mu \nu}$ tensor. However, care must be taken in placing the required Dirac matrices.

\section{FEYNMAN PROPAGATOR FOR FREE SPIN- $\frac{1}{2}$ PARTICLES}

As usual, we are able to give explicitly all Green's functions in momentum space. Let us investigate as an example the Feynman propagator $S_{F}$ which reads in momentum space

$$
S_{F}\left(x^{\prime}-x\right)=\int \frac{d^{4} p}{(2 \pi)^{4}} e^{-i p\left(x^{\prime}-x\right)} S_{F}(p)
$$

We have

$$
h^{-1} \sin \left(h p_{x^{\prime}}\right) S_{F}\left(x^{\prime}-x\right)-m S_{F}\left(x^{\prime}-x\right)=\delta^{4}\left(x^{\prime}-x\right),
$$

which has the following explicit solution in momentum space:

$$
S_{F}(p)=\frac{1}{h^{-1} \sin [(h+i \epsilon) \not p]-m} .
$$

The integration path is specified by the small quantity $+i \epsilon$. This method of defining a procedure to insure convergence in a well-defined manner has been introduced by Sommerfeld. The resulting

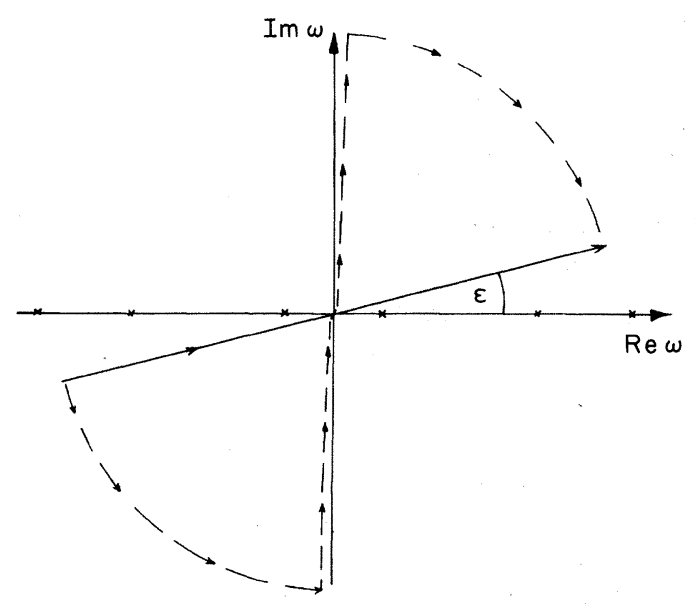

FIG. 1. The solid curve shows the integration path in the complex energy plane for the Feynman propagator; the dashed curve gives a possible deformation of the integration path which may be suitable for the complete calculation of the propagator. integration contour, when transforming back into position space, is shown in Fig. 1 for $S_{F}(p)$. The integral can be evaluated deforming the path of integration as indicated by the dashed curve in Fig. 1. Since the path of integration has been specified above, we omit the factor $+i \epsilon$ from Eq. (43).

To rationalize the denominator we multiply with $h^{-1} \sin (h \not b)+m$ and obtain

$$
\begin{aligned}
& S_{F}(p)=\frac{\left[\not p / h\left(p^{2}\right)^{1 / 2}\right] \sin \left[h\left(p^{2}\right)^{1 / 2}\right]+m}{\left(1 / h^{2}\right) \sin ^{2}\left[h\left(p^{2}\right)^{1 / 2}\right]-m^{2}} \\
& \underset{h \rightarrow 0}{\longrightarrow} \frac{\not p+m}{p^{2}-m^{2}},
\end{aligned}
$$

i.e., it goes over into the conventional form of the Feynman propagator for $h \rightarrow 0$. Incidentally, this limit again demonstrates the way one must treat $\left(p^{2}\right)^{1 / 2}$ at $p^{2}=0$.

From Eq. (44) it is obvious that there exists an infinite number of poles on the real axis. Their location is shown in Fig. 2. We shall number them with the index $n$ as indicated in the Fig. 2. Thus,

$$
\begin{aligned}
& \omega_{n}= \pm\left\{h^{-2}[\pi j+\arcsin (h m)]^{2}+k^{2}\right\}^{1 / 2} \\
& \quad \text { for } n= \pm(2 j+1), j=0,1,2, \ldots
\end{aligned}
$$

and

$$
\begin{aligned}
& \omega_{n}= \pm\left\{h^{-2}[\pi j-\arcsin (h m)]^{2}+k^{2}\right\}^{1 / 2} \\
& \text { for } n= \pm(2 j), j=1,2, \ldots .
\end{aligned}
$$

As the propagator has an infinite number of poles, it has a meromorphic expansion

$$
\begin{aligned}
& S_{F}(p)=\sum_{n=1}^{\infty} \frac{a_{n}}{\gamma \cdot p-M_{n}}, \\
& M_{n}=h^{-1}[\pi j+\arcsin (h m)] \\
& \qquad \text { for } n=2 j+1, \quad j=0,1,2, \ldots, \\
& M_{n}=h^{-1}[\pi j-\arcsin (h m)] \\
& \text { for } n=2 j, \quad j=1,2, \ldots .
\end{aligned}
$$

The coefficients $a_{n}$ are easily calculated to be

$$
a_{n}=(-)^{n+1}\left(1-h^{2} m^{2}\right)^{-1 / 2} \text { 。 }
$$

We may now perform the energy integration $\left(\tau=t-t^{\prime}\right)$,

$$
\begin{aligned}
S_{F}(\overrightarrow{\mathrm{k}}, \tau)= & \frac{1}{2 \pi} \int_{-\infty}^{+\infty} S_{F}(\overrightarrow{\mathrm{k}}, \omega) e^{i \omega \tau} d \omega \\
=\sum_{n=1}^{\infty} & \frac{(-)^{n+1}}{2 \pi}\left(1-h^{2} m^{2}\right)^{-1 / 2} \\
& \quad \times \int_{-\infty}^{+\infty} e^{i \omega \tau} \frac{\gamma \cdot p+M_{n}}{p^{2}-M_{n}^{2}+i \epsilon} d \omega .
\end{aligned}
$$

In the above equation the order of summation and integration has been exchanged. Every term in the sum may be evaluated by the usual residue method in the complex plane. This way one obtains 


$$
\begin{array}{r}
\int_{-\infty}^{+\infty} e^{i \omega \tau} \frac{\gamma \cdot p+M_{n}}{p^{2}-M_{n}^{2}+i \epsilon} d \omega=-i e^{i \omega_{+n} \tau \frac{\omega_{+n} \gamma_{0}-\overrightarrow{\mathrm{k}} \cdot \vec{\gamma}+M_{n}}{2 \omega_{+n}}} \\
\text { for } \tau<0 \\
=i e^{i \omega_{-n} \tau \frac{\omega_{-n} \gamma_{0}-\overrightarrow{\mathrm{k}} \cdot \vec{\gamma}+M_{n}}{2 \omega_{-n}}} \\
\text { for } \tau>0 .
\end{array}
$$

Formally this result may be written as an integration over the propagator (43), but this cannot be easily evaluated. We conclude this discussion by noting that as can be seen from the alternating sign $(-)^{j}$ in Eq. (47), the Green's function $S_{F}$ has self-regularizing properties as first introduced by Feynman. ${ }^{8}$

\section{GAUGE-INVARIANT NONLINEAR COUPLING WITH THE PHOTON FIELD}

To insure gauge invariance we introduce the interaction with the photon field by means of the usual minimal coupling, i.e., we replace $\not p$ by $\not p-e \not A$. Such an ansatz is manifestly gauge-invariant because our field operators are (infinite) polynomials in $\not p$. Thus an addition of $\partial \Lambda / \partial x^{\mu}$ to $A_{\mu}$ can be neutralized by a phase factor $e^{-i e \Lambda}$ in the wave function, that is,

$$
\sum a_{n}(\not p-e \not A)^{n} \psi \rightarrow \sum a_{n}(\not p-e \not A-e \gamma \cdot \partial \Lambda)^{n} e^{-i e \Lambda} \psi .
$$

Here we understand the operator $(\not p-e \not A)^{n}$ as

$$
\left(\not p-e \not A^{\prime}\right)^{n} \equiv\left\langle\left(\not p-e \not A^{\prime}\right) \cdots(\not p-e \not A)\right\rangle,
$$

where $\langle\cdots\rangle$ means that the quantity has been symmetrized in the $n$ factors of $\not p-e \not A$. Thus we have for (41)

$$
\sum a_{n}\left\langle(\not p-e \not A-e \gamma \cdot \partial \Lambda)^{n-1} \gamma^{\mu}\right\rangle\left(p_{\mu}-e A_{\mu}-e \partial_{\mu} \Lambda\right) e^{-i e \Lambda} \psi=\sum a_{n}\left\langle(\not p-e \not A-e \gamma \cdot \partial \Lambda)^{n-1} e^{-i e \Lambda}(\not p-e \not A)\right\rangle \psi .
$$

This procedure may be continued $n$ times, yielding finally the following result:

$$
\sum a_{n}(\not p-e \not A)^{n} \psi \rightarrow e^{i e \Lambda} \sum a_{n}(\not p-e \not A)^{n} \psi \text {. }
$$

This way we see that our operator $h^{-1} \sin h \not$ goes over into $h^{-1} \sin [h(\not p-e \not A)]$. Our field equation (18) reads now

$$
h^{-1} \sin [h(\not p-e \not A)] \psi(x)-m \psi(x)=0 .
$$

This equation is gauge-invariant and linear in $\psi$; however, it is nonlinear and nonlocal in $A_{\mu}$.

We will presently give a better form of the involved operator $\sin [h(\not p-e \not A)]$ so that some nonlocal properties of the above equation become more transparent. Let us therefore investigate the generalized translation operator

$$
\exp \left[i h\left(\not p-e \not A^{\prime}\right)\right]=\exp \left[-h\left(\gamma \cdot \partial+i e \not A^{\prime}\right)\right] .
$$

We define

$$
\begin{aligned}
& U(h)=\exp [i h(\not p-e \not A)], \\
& U(-h)=U^{-1}(h)=\exp [-i h(\not p-e \not A)], \\
& U_{0}(h)=e^{i h \not}, \\
& U_{0}(-h)=U_{0}^{-1}(h)=e^{-i h \not} .
\end{aligned}
$$

The operator $U(h)$ may be defined by the differential equation

$$
-i \partial_{h} U(h)=(\not p-e \not A) U(h) \text {. }
$$

We now introduce an operator $V(h)$ by

$$
\begin{aligned}
& V(h)=U_{0}^{-1}(h) U(h), \\
& V(-h)=U_{0}^{-1}(-h) U(-h) .
\end{aligned}
$$

This operator is equivalently defined by the differential equation

$$
i \partial_{h} V(h)=e U_{0}^{-1}(h) \not A U_{0}(h) V(h) \text {. }
$$

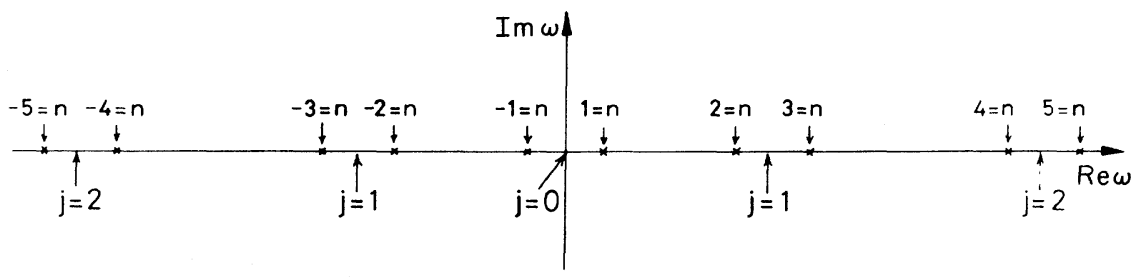

FIG. 2. Positions of the poles of the propagators. The poles are numbered from $n=-\infty$ to $n=+\infty, n \neq 0 ; n= \pm 1$ are the usual poles which remain in the limit $h \rightarrow 0$. 
This may be seen by direct differentiation of the definition (56) using (55):

$$
\begin{aligned}
& i \partial_{h} V(h)=\left[i \partial_{h} U_{0}^{-1}(h)\right] U(h)+U_{0}^{-1}(h)\left[i \partial_{h} U(h)\right] \\
& =\not p U_{0}^{-1}(h) U(h)+U_{0}^{-1}(h)(-\not p+e \not A) U(h) \\
& =U_{0}^{-1}(h) \not p U(h)-U_{0}^{-1}(h) \not p U(h) \\
& +U_{0}^{-1}(h) e \not U_{0}(h) U_{0}{ }^{-1}(h) U(h) \text {. }
\end{aligned}
$$

The solution of $(57)$ is

$$
V(h)=\exp \left(-i e \int_{0}^{h} U_{0}^{-1}\left(h^{\prime}\right) \not A(x) U_{0}\left(h^{\prime}\right) d h^{\prime}\right),
$$

where we have used $V(0)=1$. Using (56) we obtain

$$
U(h)=U_{0}(h) \exp \left(-i e \int_{0}^{h} U_{0}^{-1}\left(h^{\prime}\right) \not A(x) U_{0}\left(h^{\prime}\right) d h^{\prime}\right)
$$

Equation (59) may be written much more concisely by observing that $U_{0}(h)$ is only a translation operator acting on everything on the right-hand side with the useful property

$$
\begin{aligned}
U_{0}(h)[A(x) B(x)] & =A(x-h \gamma) B(x-h \gamma) \\
& =\left[U_{0}(h) A(x)\right]\left[U_{0}(h) B(x)\right] .
\end{aligned}
$$

In Eq. (60) we have dropped gauge-invariant terms of the form $\sigma_{\mu \nu} F^{\mu \nu}$. This means that the operator equation

$$
\exp \left(-i e \int_{0}^{h} U_{0}^{-1}\left(h^{\prime}\right) \not A(x) U_{0}\left(h^{\prime}\right) d h^{\prime}\right) \psi(x)
$$

may be written as

$$
\exp \left(-i e \int_{0}^{h} \not A^{\prime}\left(x+h^{\prime} \gamma\right) d h^{\prime}\right) \psi(x)
$$

because

$$
\begin{array}{r}
{\left[\exp \left(-i e \int_{0}^{h} U_{0}^{-1}\left(h^{\prime}\right) A^{\prime}(x) U_{0}\left(h^{\prime}\right) d h^{\prime}\right)\right] \psi(x)=\left(\sum_{n=0}^{\infty} \frac{(-i e)^{n}}{n !} \int_{0}^{h} d h^{(1)} d h^{(2)} \cdots d h^{(n)} U_{0}^{-1}\left(h^{(1)}\right) \not A(x) U_{0}\left(h^{(1)}\right)\right.} \\
\left.\times U_{0}^{-1}\left(h^{(2)}\right) \not A(x) U_{0}\left(h^{(2)}\right) \cdots U_{0}^{-1}\left(h^{(n)}\right) A^{(x)} U_{0}\left(h^{(n)}\right)\right) \psi(x) .
\end{array}
$$

Now using (60) we obtain

$$
\begin{aligned}
& =\left(\sum_{n=0}^{\infty} \frac{(-i e)^{n}}{n !} \int_{0}^{h} d h^{(1)} d h^{(2)} \cdots d h^{(n)}\left[U_{0}^{-1}\left(h^{(1)}\right) \not A(x)\right] U_{0}^{-1}\left(h^{(1)}\right) U_{0}\left(h^{(1)}\right)\left[U_{0}^{-1}\left(h^{(2)}\right) \not A(x)\right] U_{0}^{-1}\left(h^{(2)}\right) U_{0}\left(h^{(2)}\right) \cdots\right. \\
& \left.\quad \times\left[U_{0}^{-1}\left(h^{(n)}\right) \not A(x)\right] U_{0}^{-1}\left(h^{(n)}\right) U_{0}\left(h^{(n)}\right)\right) \psi(x) \\
& =\left(\sum_{n=0}^{\infty} \frac{(-i e)^{n}}{n !} \int_{0}^{h} d h^{(1)} d h^{(2)} \cdots d h^{(n)} A^{A}\left(x+h^{(1)} \gamma\right) \not A\left(x+h^{(2)} \gamma\right) \cdots \not A\left(x+h^{(n)} \gamma\right)\right) \psi(x) .
\end{aligned}
$$

Here again gauge-invariant terms of the form $\sigma_{\mu \nu} F^{\mu \nu}$ have been omitted. Using Eqs. (59), (60), and (61), we obtain

$$
\begin{aligned}
\exp \left[i h\left(\not p-e \not A^{\prime}\right)\right] \psi(x) & =e^{i h \not} \exp \left(-i e \int_{0}^{h} \not A\left(x+h^{\prime} \gamma\right) d h^{\prime}\right) \psi(x) \\
& =\exp \left(-i e \int_{0}^{h} \not A\left[x+\left(h^{\prime}-h\right) \gamma\right] d h^{\prime}\right) \psi(x-\gamma h) .
\end{aligned}
$$

Here it is understood that the $\gamma$ matrices in the argument of $\psi$ operate not only on $\psi$, as mentioned earlier, but also on the exponential before it. We shall retain the notation of (62) as no ambiguities will occur at the present. [See, however, below, Eq. (78)].

Introducing a new variable $h^{\prime \prime}=h-h^{\prime}$ we obtain

$$
\exp [i h(\not p-e \not A)] \psi(x)=\exp \left(-i e \int_{0}^{h} \not A\left(x-h^{\prime \prime} \gamma\right) d h^{\prime \prime}\right) \psi(x-h \gamma)
$$

A similar result may be obtained for the operator $e^{-i(p-e A) h}$. It is sufficient to replace $h$ by $-h$ in $(63)$ and to transfer $h^{\prime \prime}$ into $-h^{\prime}$. The result is

$$
\exp [-i h(\not p-e \not A)] \psi(x)=\exp \left(i e \int_{0}^{h} \not A\left(x+h^{\prime} \gamma\right) d h^{\prime}\right) \psi(x+h \gamma)
$$

This way the complete, gauge-invariant, Eq. (53) now reads

$$
(2 h i)^{-1}\left\{\left[\exp \left(-i e \int_{0}^{h} A^{\prime}\left(x-h^{\prime} \gamma\right) d h^{\prime}\right)\right] \psi(x-h \gamma)-\left[\exp \left(i e \int_{0}^{h} A^{\prime}\left(x+h^{\prime} \gamma\right) d h^{\prime}\right)\right] \psi(x+h \gamma)\right\}-m \psi(x)=0
$$


We now check the gauge invariance of the above equation. Note that the gauge transformations should be

$$
\begin{aligned}
& \not A(x) \rightarrow \not A(x)+\gamma \partial \Lambda(x), \\
& A^{\prime}(x+h \gamma) \rightarrow \not A(x+h \gamma)+\gamma \partial \Lambda(x+h \gamma), \\
& A^{\prime}(x-h \gamma) \rightarrow A^{\prime}(x-h \gamma)+\gamma \partial \Lambda(x-h \gamma), \\
& \psi(x) \rightarrow e^{-i e \Lambda(x)} \psi(x), \\
& \psi(x+h \gamma) \rightarrow e^{-i e \Lambda(x+h \gamma)} \psi(x+h \gamma), \\
& \psi(x-h \gamma) \rightarrow e^{-i e \Lambda(x-h \gamma)} \psi(x-h \gamma) .
\end{aligned}
$$

We need the transformation of the exponents:

$$
\int_{0}^{h} \not A\left(x-h^{\prime} \gamma\right) d h^{\prime} \rightarrow \int_{0}^{h} \not A^{\prime}\left(x-h^{\prime} \gamma\right) d h^{\prime}+\int_{0}^{h} \gamma \cdot \partial \Lambda\left(x-h^{\prime} \gamma\right) d h^{\prime} .
$$

The right-hand side may be rewritten using

$$
\begin{aligned}
\int_{0}^{h} \gamma \partial \Lambda\left(x-h^{\prime} \gamma\right) d h^{\prime} & =-\int_{0}^{h} \partial_{h^{\prime}} \Lambda\left(x-h^{\prime} \gamma\right) d h^{\prime} \\
& =\Lambda(x)-\Lambda(x-h \gamma) .
\end{aligned}
$$

Similarly we would obtain the result for the other exponent. We thus have

$$
\begin{aligned}
& \int_{0}^{h} A\left(x+h^{\prime} \gamma\right) d h^{\prime} \rightarrow \int_{0}^{h} A^{\prime}\left(x+h^{\prime} \gamma\right) d h^{\prime}-\Lambda(x)+\Lambda(x+h \gamma), \\
& \int_{0}^{h} A\left(x-h^{\prime} \gamma\right) d h^{\prime}-\int_{0}^{h} A\left(x-h^{\prime} \gamma\right)+\Lambda(x)-\Lambda(x-h \gamma) .
\end{aligned}
$$

It is now obvious that the transformation of the exponent neutralizes the transformation of the $\psi$ functions. Only an over-all phase $e^{-i e \Lambda(x)}$ remains. Explicitly (65) reads after the gauge transformation

$$
\begin{aligned}
(2 h i)^{-1} & {\left[\exp \left(-i e \int_{0}^{h} A^{\prime}\left(x-h^{\prime} \gamma\right) d h^{\prime}-i e \Lambda(x)+i e \Lambda(x-h \gamma)\right) \exp [-i e \Lambda(x-h \gamma)] \psi(x-h \gamma)\right.} \\
& \left.-\exp \left(i e \int_{0}^{h} A\left(x+h^{\prime} \gamma\right) d h^{\prime}-i e \Lambda(x)+i e \Lambda(x+h \gamma)\right) \exp [-i e \Lambda(x+h \gamma)] \psi(x+h \gamma)\right]-m \exp [-i e \Lambda(x)] \psi(x)=0
\end{aligned}
$$

This means

$[\mathrm{Eq} .(70)]=e^{-i e \Lambda(x)} \times[\mathrm{Eq} .(65)]$.

Let us build a bridge to other existing theories which are either nonlinear in the field or nonlocal in the interaction term. To do this we rewrite (65) to exhibit the interaction term:

$$
\begin{aligned}
(2 h i)^{-1}[\psi(x-h \gamma)-\psi(x+h \gamma)]-m \psi(x)= & (2 h i)^{-1}\left[1-\exp \left(-i e \int_{0}^{h} \not A\left(x-h^{\prime} \gamma\right) d h^{\prime}\right)\right] \psi(x-h \gamma) \\
& -(2 h i)^{-1}\left[1-\exp \left(i e \int_{0}^{h} A\left(x+h^{\prime} \gamma\right) d h^{\prime}\right)\right] \psi(x+h \gamma) .
\end{aligned}
$$

Let us investigate the case $h \rightarrow 0$. The left-hand side reduces to the usual Dirac equation. In order to show the behavior of the right-hand side, it must be given in powers of $h$. This can be done in two ways. The first choice is to expand the exponential leaving the integral intact, at the same time going to the limit $h \rightarrow 0$ in the argument of the fields. We obtain

$$
\begin{aligned}
(\not p-m) \psi(x) & =\frac{e}{2 h}\left(\int_{0}^{h} \not A\left(x-h^{\prime} \gamma\right) d h^{\prime}+\int_{0}^{h} \not A\left(x+h^{\prime} \gamma\right) d h^{\prime}\right) \psi(x) \\
& =\frac{e}{2 h}\left(\int_{-h}^{+h} \not A\left(x+h^{\prime} \gamma\right) d h^{\prime}\right) \psi(x) .
\end{aligned}
$$


Equation (72) is a particular approximation of Eq. (65); only the nonlocality in $\not A$ has been preserved; the nonlinearity in $\not A$ and the nonlocality in $\psi(x)$ have been neglected. The integral in Eq. (72) may be evaluated completely:

$$
\begin{aligned}
\frac{e}{2 h} \int_{-h}^{+h} \not A\left(x+h^{\prime} \dot{\gamma}\right) d h^{\prime} & =\frac{e}{2 h} \int_{-h}^{+h} \sum_{n=0}^{\infty} \frac{(\gamma \cdot \partial)^{n}}{n !} h^{\prime n} \not A(x) d h^{\prime} \\
& =\frac{e}{2 h} \sum_{n=0}^{\infty} \frac{(\gamma \cdot \partial)^{n}}{(n+1) !} \not A(x)\left[h^{n+1}-(-h)^{n+1}\right] \\
& =e \sum_{n=0}^{\infty} \frac{(\gamma \cdot \partial)^{2 n}}{(2 n+1) !} h^{2 n} \not A(x) \\
& =e \frac{\sin (h \not p)}{h \not p} \not A(x) \equiv e \frac{\sin \left[h(-\square)^{1 / 2}\right]}{h(-\square)^{1 / 2}} \not A(x) .
\end{aligned}
$$

Another possible procedure is to perform an approximate integration of the exponents in (65). Using the Eq. (73) the following result is obtained:

$$
\begin{aligned}
(\not p-m) \psi(x) & =\frac{1}{2 h i}\left(e^{+i e h A(x)}-e^{-i e h A(x)}\right) \psi(x) \\
& =h^{-1} \sin [h e \not A(x)] \psi(x) .
\end{aligned}
$$

Only nonlinear terms in $\not A$ have been retained. Equation (74) is local.

Both these approximate equations [Eqs. (72) and (74)] are not gauge-invariant. This can be traced to the inconsistent way in which the limit $h \rightarrow 0$ has been performed. However, going to the full limit $h \rightarrow 0$ we of course obtain the inhomogeneous Dirac equation for both (72) and (74). This shows in which way the generalizations of the Dirac equation such as either Eq. (72) or Eq. (74) are inconsistent. They may be fused into a consistent gauge-invariant theory involving both nonlinearities and nonlocalities.

Now we will derive a divergence-free four-vector for the coupled equations which in the limit $h \rightarrow 0$ reduces to the Dirac-current four-vector. We begin with the field equations $\psi$ and $\bar{\psi}$. First we derive an equation of motion for the adjoint fields. We take $A_{\mu}$ to be real, i.e., to be a linear combination of both positive and negative frequencies. We recall $\gamma^{+}=\gamma_{0} \gamma \gamma_{0}$. Another useful operator equation is

$$
e^{\gamma_{0} x \gamma_{0}}=\gamma_{0} e^{x} \gamma_{0} .
$$

The adjoint of Eq. (65) is (recall that the $A_{\mu}$ field is always real)

$$
(2 h i)^{-1}\left[\bar{\psi}(x-h \gamma) \exp \left(i e \int_{0}^{h} \not A\left(x-h^{\prime} \gamma\right) d h^{\prime}\right)-\bar{\psi}(x+h \gamma) \exp \left(-i e \int_{0}^{h} \not A\left(x+h^{\prime} \gamma\right) d h^{\prime}\right)\right]-m \bar{\psi}(x)=0 .
$$

We multiply Eq. (65) by $\bar{\psi}(x)$ from the right, Eq. (76) by $\psi(x)$ from the left, and subtract:

$$
\begin{aligned}
\bar{\psi}(x) \exp \left(-i e \int_{0}^{h} \not A\left(x-h^{\prime} \gamma\right) d h^{\prime}\right) & \psi(x-h \gamma)-\bar{\psi}(x) \exp \left(i e \int_{0}^{h} A^{\prime}\left(x+h^{\prime} \gamma\right) d h^{\prime}\right) \psi(x+h \gamma) \\
& +\bar{\psi}(x-h \gamma) \exp \left(i e \int_{0}^{h} \not A\left(x-h^{\prime} \gamma\right) d h^{\prime}\right) \psi(x)-\bar{\psi}(x+h \gamma) \exp \left(-i e \int_{0}^{h} A^{\prime}\left(x+h^{\prime} \gamma\right) d h^{\prime}\right) \psi(x)=0 .
\end{aligned}
$$

We may write the first and the last term in Eq. (77) together after we have used (64). Similarly we treat the middle terms of (77) and obtain [recall the remarks after Eq. (62)]

$$
\bar{\psi}(x)[\exp (-h \gamma \cdot \vec{\partial})-\exp (h \gamma \cdot \vec{\partial})] \chi(x)-\bar{\psi}(x)[\exp (h \gamma \cdot \vec{\partial})-\exp (-h \gamma \cdot \vec{\partial})] \xi(x)=0,
$$

with

$$
\begin{aligned}
& \chi(x)=\exp \left(-i e \int_{0}^{h} \not A\left(x+h^{\prime} \gamma\right) d h^{\prime}\right) \psi(x), \\
& \xi(x)=\exp \left(i e \int_{0}^{h} \not A\left(x-h^{\prime} \gamma\right) d h^{\prime}\right) \psi(x) .
\end{aligned}
$$

We now want to write Eq. (78) as a divergence of a.four-vector. To do so we investigate the operators

$$
O^{-}=\exp (-h \gamma \cdot \vec{\partial})-\exp (+h \gamma \cdot \vec{\partial}) \text {, }
$$




$$
O^{+}=\exp (h \gamma \cdot \vec{\partial})-\exp (-h \gamma \cdot \overleftarrow{\partial})
$$

more closely. We first separate the even and odd terms in the expansion of the exponentials:

$$
\begin{aligned}
O^{-} & =-\sum_{n=0}^{\infty} \frac{(h \gamma \cdot \vec{\partial})^{2 n+1}+(h \gamma \cdot \vec{\partial})^{2 n+1}}{(2 n+1) !}+\sum_{n=0}^{\infty} \frac{(h \gamma \cdot \vec{\partial})^{2 n}-(h \gamma \cdot \vec{\partial})^{2 n}}{(2 n) !} \\
& \equiv \Omega^{\text {even }}-\Omega^{\text {odd }}, \\
O^{+} & =\sum_{n=0}^{\infty} \frac{(h \gamma \cdot \vec{\partial})^{2 n}-(h \gamma \cdot \overleftarrow{\partial})^{2 n}}{(2 n) !}+\sum_{n=0}^{\infty} \frac{(h \gamma \cdot \vec{\partial})^{2 n+1}+(h \gamma \cdot \vec{\partial})^{2 n+1}}{(2 n+1) !} \\
& \equiv \Omega^{\text {even }}+\Omega^{\text {odd }} .
\end{aligned}
$$

We now note the following identities:

$$
\begin{aligned}
A(x) \Omega^{\text {odd }} B(x)= & \partial_{\mu}\left[A(x)\left(\sum_{n=0}^{\infty} \sum_{k=0}^{2 n} \frac{(-)^{n}}{(2 n+1) !} h^{2 n}(\gamma \cdot \overleftarrow{\partial})^{2 n-k} \gamma^{\mu}(\gamma \cdot \vec{\partial})^{k}\right) B(x)\right] \\
& \equiv \partial_{\mu}\left[A(x) \vec{J}^{\mu} B(x)\right], \\
A(x) \Omega^{\text {even }} B(x) & =\partial_{\mu}\left[A(x)\left(\sum_{n=0}^{\infty} \sum_{k=0}^{2 n}\left(\vec{\partial}^{\mu}-\overleftarrow{\partial}^{\mu}\right) \frac{h^{2 n}}{(2 n) !} \overleftarrow{\square}^{k} \vec{\square}^{n-k-1}\right) B(x)\right] \\
& \equiv \partial_{\mu}\left[A(x) \vec{K}^{\mu} B(x)\right] .
\end{aligned}
$$

They can be proven by direct differentiation. We obtain finally from Eq. (78), inserting all necessary factors to achieve Dirac's current in the case $h \rightarrow 0$,

$$
j_{\mu}=\frac{1}{2} e\left[\bar{\psi}(x)\left\{\vec{J}_{\mu}+\ddot{K}_{\mu}\right\} \xi(x)+\bar{\psi}(x)\left\{\vec{J}_{\mu}-\ddot{K}_{\mu}\right\} \chi(x)\right] .
$$

To complete the photon-electron interaction we add a possible generalization of the free equations for the vector potentials. According to Eq. (5b) we assume the following form:

$$
-h^{-2}\left[A_{\mu}(x+h \gamma)+A_{\mu}(x-h \gamma)-2 A_{\mu}(x)\right]=0 .
$$

Being a generalization of the equation $\square A_{\mu}=0$ the Eq. (84) is valid only in the Lorentz gauge. By a proper definition of the $\gamma$ matrices this equation may be made to be gauge-invariant. ${ }^{9}$ We shall not consider this question here and restrict ourselves to the Lorentz gauge. In this case the coupled equations for the $A_{\mu}$ field read

$$
-h^{-2}\left[A_{\mu}(x+h \gamma)+A_{\mu}(x-h \gamma)-2 A_{\mu}(x)\right]=j_{\mu},
$$

where $j_{\mu}$ is given by (83).

\section{SUMMARY AND OUTLOOK}

In this paper we have described a classical theory of a spin- $\frac{1}{2}$ field interacting with a vector field, which is nonlocal in the sense that the field equations connect the field at a given point $x$ with the fields of such points which are separated by a hypercomplex distance of order $h$ from the point $x$. Hence one could call it a hyperrelativistic field theory. The field equations are local as far as the Minkowski coordinate $x$ is concerned. The theory is Lorentz- and gauge-invariant, and it reduces to the Dirac theory in the limit $h \rightarrow 0$. A particular form of the Lagrangian was considered. Other forms are possible and perhaps preferable. For example, one may exponentiate also the mass term, i.e., use as the basic form the operator $\exp \left[i h\left(\gamma \cdot p+\gamma_{5} m\right)\right]$ in Eq. (16). Also, one may generalize the Lagrangian by writing

$$
\mathcal{L}^{\prime}=\int_{-\infty}^{+\infty} d h^{\prime} g\left(h^{\prime}\right) \bar{\psi}(x) e^{i h^{\prime} \gamma \cdot p} \psi(x),
$$

where a suitable $g\left(h^{\prime}\right)$ is used. Equation (13) is obtained with the choice

$$
g\left(h^{\prime}\right)=(i / 2 h)\left[\delta\left(h+h^{\prime}\right)-\delta\left(h-h^{\prime}\right)\right]-m \delta\left(h^{\prime}\right) .
$$

Since the field theory is equivalent to a theory containing infinite-order differentiations in Minkowski space, it is formally equivalent to a theory which describes a system of particles with an infinite discrete-mass spectrum. By the choice of the phase of the nonlocality parameter $h$ they can be made to have real masses. [Alternatively, they can be made to be tachyons (see Appendix A).] Therefore the Green's functions have self-regulatory properties; they can be considered to be rep- 
resented formally by an infinite sum over "elementary" graphs. Any graph computed with propagators of the kind of (44) has a finite value. Finally, explicit expressions were obtained for the current four-vector and the energy-momentum tensor of the theory.

Since in a theory with infinite derivatives it is difficult to write expressions for the canonically conjugate fields, i.e., the momentum fields, all manipulations were carried out formally without their use in terms of operator equations involving integrations over variables belonging to the Minkowski-Clifford space. This is important in view of the next step, viz., the quantization of the fields; that will also have to be done without the explicit use of momentum fields. It will be performed in terms of the conserved quantities of the theory, i.e., in terms of the current four-vector and the energy-momentum tensor. As the required mathematics is not too familiar all results were checked out by direct calculation.

We shall discuss the questions connected with the quantization of the theory in detail in a forthcoming paper. Nevertheless, it may be instructive to discuss some of the qualitative aspects already at this point. To begin, let us discuss the vacuum polarization. Since the current density is different from that of the Dirac theory, the vertex function obtains also a new form. Therefore the questions concerning the convergence of the Feynman perturbation expansion must be reexamined. This can be done by noting that the requirement of gauge invariance expressed in the form $p_{\mu} j^{\mu}=0$ can be used to estimate the asymptotic behavior of $j$ for large $p$, namely, the charge-conservation condition can be fulfilled identically only if the expression $p_{\mu} j^{\mu}$ for mally reduces to the equations of motion. Thus asymptotically in momentum space there must hold

$$
p j \sim 1 / G \text {. }
$$

This must give rise to a quadratic divergence of the lowest-order vacuum-polarization graph as it is of the form

$$
\int d^{4} p j G j G
$$

This is true for any gauge-invariant theory, e.g., for the usual QED. These considerations show clearly the origin of the convergence of field theories where the question of gauge invariance has not been considered. ${ }^{10}$ Examples of such theories are Eqs. (72) and (74), where the gauge invariance of Eq. (71) has been lost because of an improper limiting procedure. In the present theory the convergence comes about by virtue of the fact that the full gauge-invariant graph having one virtual elec- tron-positron pair is, in fact, described by a superpropagator in which in addition to the pair an arbitrary number of photons is exchanged between the two vertices. Each of these graphs by itself is not gauge-invariant. In particular, the lowest of the graphs, in which no photon is exchanged between the vertices, does not fulfil (88). Thus here (89) can be, and in fact is, convergent. The graphs in which in addition photons are exchanged between the vertices have additional propagators, and are also finite.

In the present paper we have treated explicitly only the case of "electrons" and "photons." No difficulties arise when introducing hadrons. Thus, one could describe the meson-nucleon interaction by using the replacement $A_{\mu} \rightarrow \partial_{\mu} \varphi$, of course augmented by the necessary isospin, etc. couplings.

All these fields and coupling constants are unrenormalized quantities.

Similarly one can consider the weak interactions. In fact, they are of particular interest in the present context since the weak coupling constant has the dimension (length) ${ }^{2}$. One may thus suspect

$$
h^{2}=\text { const } \times G,
$$

where the constant depends on the details of the manner in which one chooses to write the weakinteraction Lagrangian. At any rate, this would imply a value for $h^{-1}$ of the order of $300 \mathrm{GeV}$. The weak interactions this way could be said to be responsible for the convergence of strong and electromagnetic field theory - a frequently mentioned speculation. The unphysical result of the usual theory that the weak processes, e.g., neutrino scattering, violate unitarity at $E \gtrsim 300 \mathrm{GeV}$ presumably will be eliminated by higher-order rescattering via "weak" vertices which at that energy have, in fact, become strong. In conventional theory the relevant graphs, which are essentially vacuum-polarization graphs, diverge quadratically, while here they are finite.

Having finite solutions the theory will force a rethinking of the question of physical interpretation. To begin with, the parameters of the theory, the masses and the coupling constants appearing in the Lagrangian, are bare quantities. The clothed particles must be obtained from solving the coupled equations, i.e., one has to compute the finite renormalizations. This way one will obtain explicitly composite structures for the particles; they will contain an arbitrary number of bare particles they will exhibit parton properties. ${ }^{11}$

As a final point we mention that in higher-order corrections the present theory will yield somewhat different values than the renormalized QED. (The lowest-order graphs are insensitive to the kind of 
modifications of QED effected by the present theory. ${ }^{12}$ ) The expected magnitude of these deviations will be of the order $m_{e} h$ ( $m_{e}$ is the electron mass). For example, the value of $g-2$ for the electron would be changed by $(\alpha / 2 \pi) m_{e} h \sim 10^{-6}(\alpha / 2 \pi)$ which is about at the limit of the present experimental and theoretical accuracy. ${ }^{13}$ An improvement of only an order of magnitude in the accuracy of the experimental number will be required to show deviations of such a magnitude from the predictions of QED. Needless to say, these estimates are highly speculative; in particular, the assumption $h^{2}=G$ is purely hypothetical.

To summarize the outlook, we believe that the present theory can give a good starting point for the investigation of many unsolved questions in that it provides a possible framework in which to perform actual calculations.

\section{APPENDIX}

In this appendix we investigate the properties of the theory given by Eq. (18) with imaginary $h$. We introduce a new real parameter $l$ by

$$
l=i h
$$

and obtain from Eq. (18)

$$
\left[l^{-1} \sinh (l \not p)-\dot{m}\right] \psi(x)=0 \text {. }
$$

The poles of the Green's function are easily found to be defined by

$$
l^{-2} \sinh ^{2}\left[l\left(p^{2}\right)^{1 / 2}\right]=m^{2} .
$$

We obtain in analogy to Eqs. (45) and (46b)

$$
\begin{aligned}
M_{n}= & \pm l^{-1}[i \pi j+\operatorname{arcsinh}(l m)] \\
& \quad \text { for } n= \pm(2 j+1), j=0,1,2, \ldots, \\
M_{n}= \pm l^{-1}[i \pi j-\operatorname{arcsinh}(l m)] & \\
& \text { for } n= \pm(2 j), j=1,2, \ldots,
\end{aligned}
$$

and we find the energy poles at

$$
\begin{aligned}
& \omega_{n}=+\left(M_{n}^{2}+k^{2}\right)^{1 / 2} \text { for } n>0, \\
& \omega_{n}=-\left(M_{n}^{2}+k^{2}\right)^{1 / 2} \text { for } n<0 .
\end{aligned}
$$

It is obvious that the values of $\omega_{n}$ are complex because the masses $M_{n}$ are complex. For $j \neq 0, M_{n}$ is almost completely determined by the term $i \pi j / l$. In Fig. 3(a) the masses and in Fig. 3(b) the poles in the complex energy plane are shown.

All formulas derived so far can be rewritten us-
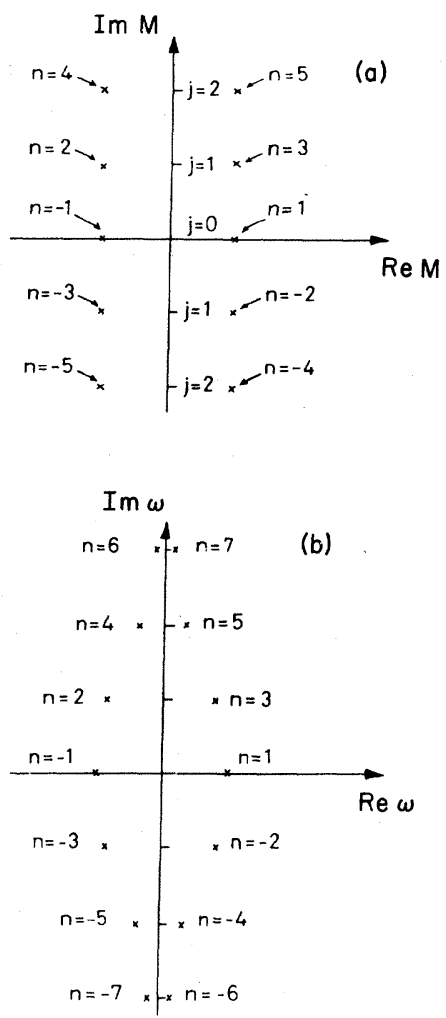

FIG. 3. The singularities of a theory with purely imaginary $h$ : (a) The complex masses of the noncausal theory. Only the masses $n= \pm 1$ are real. The other masses correspond to tachyons. (b) The poles of the propagator in the complex energy plane.

ing Eq. (A1). We quote here only the result for the meromorphic expansion of the Feynman propagator to stress the noncausal behavior of such a theory. We have

$$
S_{F}^{\operatorname{im}}(p)=\sum_{\substack{n=-\infty \\ n \neq 0}}^{+\infty} \frac{(-)^{j}\left(1+l^{2} m^{2}\right)^{-1 / 2}}{\gamma \cdot p-M_{n}+i \epsilon} .
$$

The energy integration [Eq. (48)] can be carried out and the result is completely different from Eq. (49) since two corresponding poles are always together in the upper $(n>1)$ or lower $(n<1)$ part of the energy plane [see Fig. 3(b)]. (The poles $n= \pm 1$ lie as usually on the real axis.) As is well known, ${ }^{14}$ the resulting Feynman propagator is noncausal.
*Work supported in part by Deutsche Forschungsgemeinschaft and by Bundesministerium für Bildung und Wissenschaft.

${ }^{1}$ W. Heisenberg, Einführung in die einheitliche Feld- theorie der Elementarteilchen (Stuttgart, 1967).

${ }^{2}$ See, for example, R. Dashen, Phys. Rev. 183, 1245 (1969).

${ }^{3}$ A. Salam, in Proceedings of the 1971 Coral Gables 
Conference on Fundamental Interactions at High Energy, edited by M. Dal Cin, G. J. Iverson, and A. Perlmutter (Gordon and Breach, New York, 1971).

${ }^{4}$ W. K. Clifford, Am. J. Math. 1, 350 (1878).

${ }^{5}$ J. D. Bjorken and S. D. Drell, Relativistic Quantum Fields (McGraw-Hill, New York, 1965).

${ }^{6}$ This expansion has been already given by $\mathrm{O}$. Hellman, Nucl. Phys. 52, 609 (1964).

${ }^{7} \mathrm{~A}$ similar procedure may be found also in Jan Rzewuski, Field Theory (ILIFE, London, 1969), Vol. II.

${ }^{8}$ R. P. Feynman; Phys. Rev. 74, 939 (1948); 76, 769 (1949).

${ }^{9}$ R. W. Hayward, private communication.
${ }^{10}$ See, for example, D. L. Nordstrom, Phys. Rev. D $\underline{4}$, 1611 (1971).

${ }^{11} \mathrm{M}$. Danos, in High Energy Physics and Nuclear Structure, edited by S. Devons (Plenum, New York, 1970), p. 811.

${ }^{12}$ N. M. Kroll, Nuovo Cimento 45A, 65 (1966).

${ }^{13}$ S. J. Brodsky, in Precision Measurement and Fundamental Constants, National Bureau of Standards Special Publication No. 343 , edited by D. N. Langenberg and

B. N. Taylor (National Bureau of Standards, Washington, D. C., 1971), p. 297.

${ }^{14}$ See, for example, P. Roman, Introduction to Quantum Field Theory (Wiley, New York, 1969).

\title{
Second-Order Radiative Corrections to the Triangle Anomaly. II*
}

\author{
Kimball A. Milton, Wu-yang Tsai, and Lester L. DeRaad, Jr. \\ Department of Physics, University of California, Los Angeles, California 90024
} (Received 10 May 1972)

\begin{abstract}
In a preceding paper, we have calculated the radiative correction to the "triangle anomaly, " using source-theoretical techniques. Here we apply the same methods to a different causal arrangement, and obtain the same result, giving a total anomaly through order $\alpha$ proportional to $1+\alpha / 2 \pi$. In order to obtain this result, we found it necessary to compute (for $m_{\pi}=0$ ) the first radiative corrections to the $\pi^{0}$ photoproduction amplitudes, which are presented here for the first time.
\end{abstract}

\section{INTRODUCTION}

In a preceding paper ${ }^{1}$ we have shown that the low-energy theorem for pion decay is subject to radiative corrections, as expressed by the factor

$$
\tilde{I}=1+\alpha / 2 \pi+O\left(\alpha^{2}\right) .
$$

We showed there that this result is not in formal disagreement with that of other authors, since the low-energy theorem is necessarily expressed in terms of the $\pi N \bar{N}$ coupling constant at zero momentum transfer squared.

The main purpose of this paper is to offer support to the calculational techniques used in I. One of the important aspects of source theory is the application of internal consistency requirements by considering a given process under different causal situations. The calculation of I was the first not to employ the techniques of operator field theory, so it would not seem inappropriate to offer a second calculation using our methods. Whereas in I spectral forms in terms of the pion momen- tum were obtained, we will here derive spectral forms in terms of the momentum of one of the photons. Since the anomaly is defined for all momenta equal to zero, without loss of generality we may set $m_{\pi}=0$ throughout.

The second purpose of this paper is to present the lowest-order radiative corrections to the $\pi^{0}$ photoproduction amplitudes. Here we will consider the case $m_{\pi}=0$ : This is a common approximation when performing calculations involving pions, and is here done for the obvious reason of kinematic simplicity; certainly this will suffice, for our calculation of the anomaly. To our knowledge, those amplitudes have not been calculated before.

To provide a basis for the radiative corrections to the anomaly, we will here compute the lowestorder triangle process, which is defined by the causal stipulations of Fig. 1. The vacuum amplitude describing the production of two fermions by an extended photon source, and their subsequent annihilation into a photon and a pion, is [ $\mathrm{cf}$. Eq. (I4) $]\left(k_{1}+q=Q\right)$ 